\title{
Impact of mechanical deformation on space charge in XLPE
}

\author{
G. Chen ${ }^{*}$ and M. R. Kamaruzzaman
}

\author{
School of Electronics and Computer Science \\ University of Southampton, \\ Southampton, SO17 1BJ, United Kingdom \\ * E-mail : gc@ecs.soton.ac.uk
}

\begin{abstract}
In this paper we report the effect of mechanical deformation on space charge dynamics in crosslinked polyethylene. Thin films were peeled from a $66 \mathrm{kV}$ commercial XLPE cable. Space charge measurements under dc electric fields have been monitored using the pulsed electroacoustic (PEA) technique. It has been found that charge dynamics in deformed XLPE are different from that from undeformed XLPE at lower voltages. At low applied electric field, space charge is dominated by heterocharge in the deformed XLPE while homocharge governs charge formation in undeformed XLPE. However, at high electric field, there is no significant difference between them. Space charge is dominated by homocharge in both deformed and undeformed XLPE. The results indicated that through deformation, molecular chains rearrange so small molecules such as crosslinking by-products, initially being trapped, can be released. Under the influence of the applied electric field they can be ionised to form heterocharge in the sample.
\end{abstract}

\section{INTRODUCTION}

Power cables are an essential part of power transmission and distribution systems and their performance is a great concern to the reliability of the systems. In addition to their requirements of electrical performance, power cables experience various mechanical stresses during their manufacture, transportation, layout and operation. This may affect the electrical performance of power cables in service. Consequently, there are considerable studies in last few decades that explore the influence of mechanical stress on the dielectric breakdown strength [1-2], on the propagation of electrical tree [3] of crosslinked polyethylene (XLPE) power cable insulation. Ilstead et al [1] showed that a significant reduction in ac breakdown strength occurred when XLPE cable samples were subjected to $21 \%$ strain. Our early research [3] indicated that electrical tree growing characteristics have been significantly changed when mechanical stress is introduced. However, a complete description of the phenomena is still beyond reach and more research is necessary.

In the present paper, the influence of mechanical stress on space charge formation in XLPE material is investigated. $40 \%$ strain has been adopted and space charge formation at $25 \mathrm{kV} / \mathrm{mm}$ and $50 \mathrm{kV} / \mathrm{mm}$ has been measured using the pulsed electroacoustic technique (PEA). To eliminate the effect of possible broken chains and generation of smaller molecules on space charge formation, space charge in additive-free low density polyethylene film has also been studied.

\section{EXPERIMENTAL DETAILS}

\section{Sample details}

XLPE samples were prepared from a commercial $66 \mathrm{kV}$ XLPE power cable. A section of 70 to $100 \mathrm{~mm}$ was removed from the cable and thin tapes were peeled from the section. The thickness of the tape was controlled in the range from 100 to $200 \mu \mathrm{m}$, For comparison test, similar thickness of low density polyethylene (LDPE) samples were purchased commercially. To avoid complication due to additives, additive-free LDPE was used in the present study.

There are several forms of deformations due to various stresses experienced by power cables. In the present paper the study was concentrated on tensile stress. The tensile stress was applied to the sample using the INSTRON. A total extension of $20 \mathrm{~mm}$ from $50 \mathrm{~mm}$ (40\% strain) sample length provides sufficient strain information to investigate mechanical deformation of polymeric material on space charge. A further higher strain percentage was tried however it was found out that at $90 \%$ of strain, the sample surface started to wrinkle significantly and make space charge measurement difficult.

\section{PEA system}

The PEA technique was chosen to detect space charge in samples due to its simplicity in structure, low cost and easy to implement. A schematic diagram for a conventional PEA system is shown in Fig.1. A high voltage pulse with $5 \mathrm{~ns}$ length is applied to the sample sandwiched between the two electrodes. The pulse electric field produced interacts with charge layers, generating an electric force which displays charge. The consequence is the formation of pulsed acoustic waves in correspondence of each charge layer with respect to neutrality. The resultant acoustic signals are detected by a piezo-electric transducer so that the charge distribution in the sample under test can be obtained from the output voltage profile of the transducer. The 
electric signal obtained in time domain represents the charge distribution. The analysis of space charge profiles is restricted to one dimension.
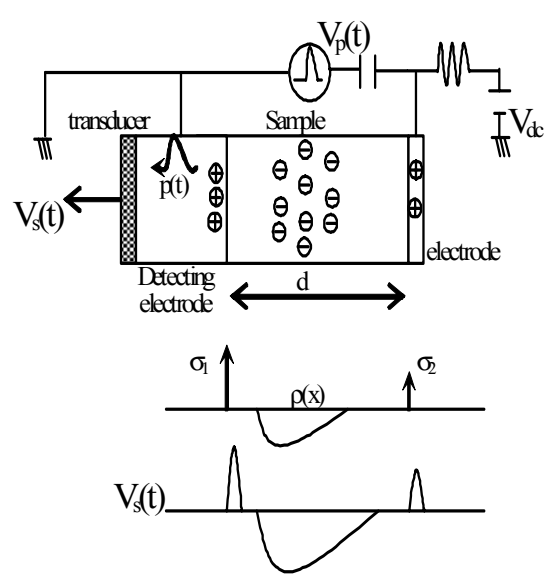

Figure 1 Schematic diagram of PEA technique.

A thin semiconducting film (LDPE loaded with carbon black) was attached to the top copper electrode to improve acoustic matching. A very thin layer of silicone oil was applied to the interface between electrodes and sample to achieve a better acoustic transmission. Quantitative charge analysis needs to calibrate the system and this is typically done by applying a small voltage across the sample to generate a known charge density on the two surfaces at the electrodes. The procedure is termed as calibration and more details can be found in our published work [4].

To observe space charge in the samples, two voltage levels were used in the present study. A voltage profile is shown in Fig. 2.

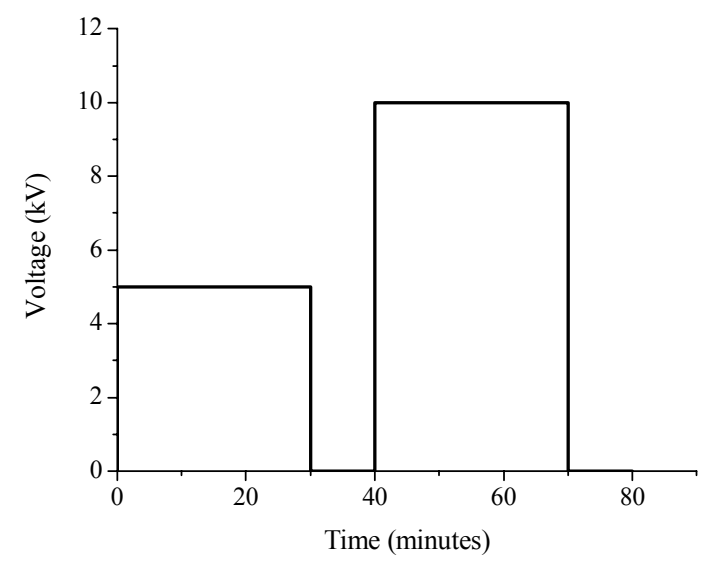

Fig. 2 Voltage profile of space charge measurements.

\section{RESULTS AND DISCUSSION}

\section{Space charge in undeformed XLPE}

Fig. 3 shows space charge profiles after the removal of the applied voltages. The electrode polarities during stressing period are also illustrated in the figure. After 5 $\mathrm{kV}$ for 30 minutes, negative charge is observed in the region adjacent to the cathode while small amount negative charge is present close to the anode as well. It is believed that the negative charge close to the cathode is due to charge injection from the cathode. The applied field is around $25 \mathrm{kV} / \mathrm{mm}$ which is above the threshold field value for charge injection for XLPE [5]. The small amount negative charge close to the anode may be due to ionisation from crosslinking by-products. At $10 \mathrm{kV}$, charge distribution after the removal of the applied voltage is very different from $5 \mathrm{kV}$. Instead of negative charge, positive charge is formed adjacent to the cathode and the negative charge adjacent to the anode shows a significant increase. There is small amount of negative charge in the bulk. It seems that heterocharges formed closed to the electrodes are due to ionisation of crosslinking by-products. Under high electric field the ionisation will be more and ionised ions move towards the opposite electrodes under the influence of the applied field. The bulk negative charge is probably the result of charge injection from the electrode.

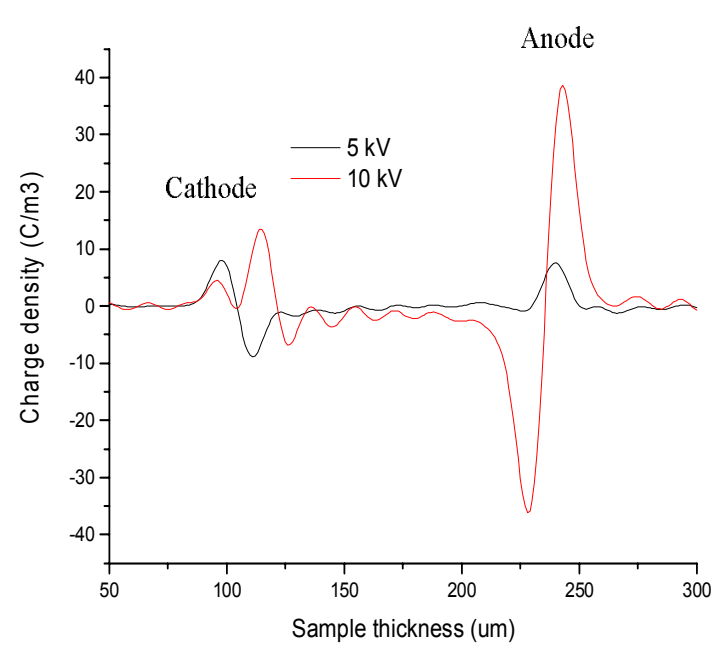

Fig. 3 Space charge in undeformed XLPE after the removal of the applied voltages.

\section{Space charge in deformed XLPE}

Space charge profiles after the removal of the applied voltages in deformed XLPE sample is shown in Fig. 4. AT $5 \mathrm{kV}$ there are negative charges formed adjacent to both electrodes. The magnitude of negative charge close to the anode is higher than that in undeformed sample while the magnitude of the negative charge close to the cathode is lower. This may indicate that more ionisable crosslinking by-products are available in the deformed sample. After the removal of $10 \mathrm{kV}$, the positive charge observed close to the cathode and the negative charge is significant. The negative charge across the bulk is due to charge injection from the cathode. 


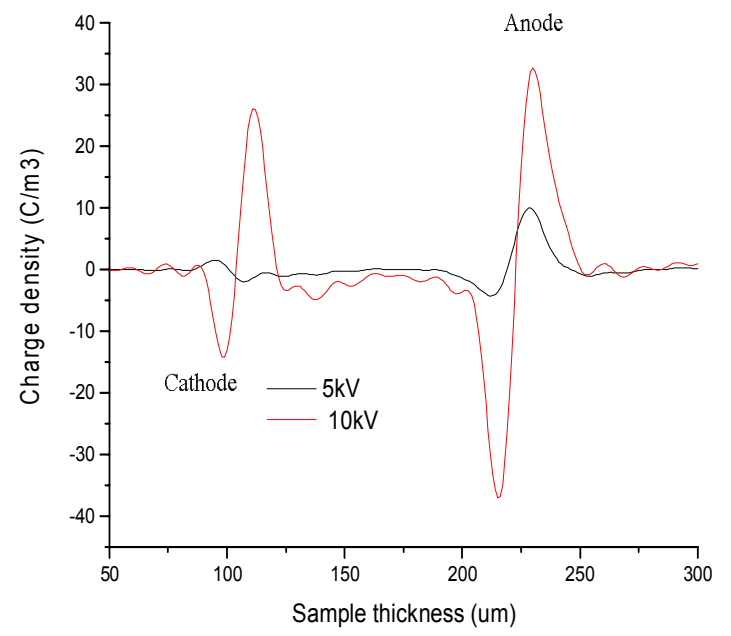

Fig. 4 Space charge in the deformed XLPE after the removal of the applied voltages.

Comparing the results from the deformed and undeformed XLPE samples, there is some obvious features showing their differences. It is noticed that negative heterocharges close to the anode are greater after the sample is deformed even though at a low applied voltage. At high applied voltage, the positive charge close to the cathode is much higher than that in the undeformed XLPE sample while the negative charge adjacent to the anode is similar. The XLPE cable used in the present research is an unconditional cable and has been manufactured for 3 years. Some of crosslinking by-products may undergo natural diffusion process and while the rest remain in the material. Under the influence of the electric field, ionisation may take place. The ionised species will move towards the opposite electrode to form heterocharges. There are two factors that may affect the amount of charge formed in the material and they are the number of ionisable species and electric field strength. At $5 \mathrm{kV}$ the amount of ionised species will be small due to low field strength. This can be seen in both deformed and undeformed samples. The slightly increase in negative charge close to the anode in the deformed sample is attributed to the increase in ionisable species. At $10 \mathrm{kV}$ the electric field strength is sufficiently high to cause more ionisation of the ionisable species. However, in the deformed XLPE sample it seems that there are more ionisable species than in the undeformed sample as there are more positive charges in the region close to the cathode. To explain the more ionisable species in the deformed XLPE sample the following is proposed. Some of the crosslinking by-products were locked in molecular chains of XLPE. Under the normal condition they are not easy to move and difficult to ionise. When the tensile stress is applied molecular chains start to move. The movement of molecular chains may release these locked by-products. Consequently the released byproducts form part of the ionisable species.
Alternatively, deformation of XLPE sample may lead to broken chains or even smaller molecules. The broken chains and smaller molecules may be ionised under the electric field. To eliminate this proposal, space charge profiles in additive free LDPE films were also conducted. LDPE sample was deformed exactly same as the XLPE sample. The voltage profile was applied to LDPE samples. Fig. 5 shows the space charge profile after the removal of $10 \mathrm{kV}$.

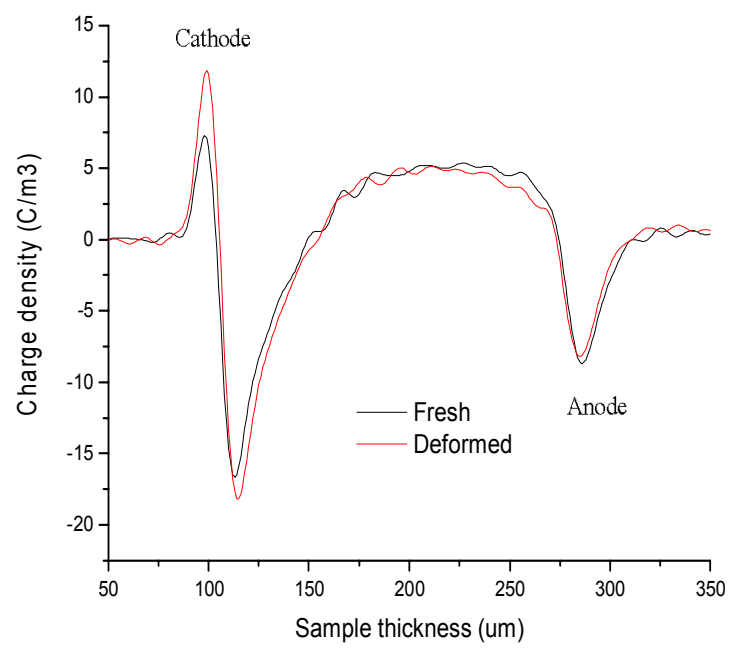

Fig. 5 Space charge profiles in LDPE after the removal of $10 \mathrm{kV}$.

It can be seen that there is little difference between deformed and fresh LDPE. Homecharges are formed and they can be attributed to the charge injection from both anode and cathode. Since there is no ionisable species in the material therefore no hetercharge is expected from the fresh LDPE. If the deformation can produce smaller molecules and broken chains then it may be possible to observe heterocharge at high field. However, the space charge profile from deformed LDPE shows no heterocharge. Therefore, the alternative explanation is not valid.

\section{CONCLUSION}

Mechanical impact on space charge formation in XLPE sample taken from a commercial power cable has been assessed using the PEA technique. By comparing the results obtained from various samples, the following conclusions may be drawn.

Heterocharge is observed in both undeformed and deformed XLPE samples. Formation of charge can be attributed to the crosslinking by-products remained in the cable. Deformation causes the molecular chains to move. The extra heterocharge observed in the deformed XLPE sample is believed to due to release of crosslinking by-products trapped within the molecular chains. 


\section{REFERENCES}

[1] E. Ildstad, S.T. Hagen, Electrical Treeing and breakdown of Mechanically strained XLPE Cable Insulation, IEEE Symposium on Electrical Insulation 2004

[2] C. Martin, The Impact of Mechanical Stress on the Integrity of XLPE Cable, PhD thesis University of Southampton, 2004

[3] X. Zheng, G. Chen, A. E. Davies, S. J. Sutton and S. G. Swingler, "Mechanical stress and voltage frequency on electrical tree in XLPE”, CEIDP, Mexico, pp.955-958, 2002.

[4] G. Chen, Y.L. Chong and M. Fu, Calibration of the Pulsed Electroacoustic Technique in the Presence of Trapped Charge. Measurement Science and Technology 17(7) pp. 1974-1980, 2006.

[5] G. C. Montanari, G. Mazzanti, F. Palmieri, A. Moton, G. Perego and S. Serra, "Space-charge trapping and conduction in LDPE, HDPE and XLPE", J. Phys. D: Appl. Phys. Vol. 34, pp. 29022911, 2001 